\title{
USING FIBRE OPTIC CABLES TO DELIVER INTELLIGENT TRAFFIC MANAGEMENT IN SMART CITIES
}

\author{
A.J. Hall ${ }^{*}$ and $\operatorname{Dr}$ C. Minto \\ OptaSense, Cody Technology Park, Farnborough, Hants, GU14 OLZ, UK. \\ *Corresponding author
}

\begin{abstract}
As the demand for mobility grows, the stresses placed on city road networks intensifies. Optimal use of road infrastructure is vital to managing the impact of rapidly increasing traffic volumes and minimising congestion and journey times. Imagine monitoring traffic effectively by using existing fibre optic cables buried around the system. Distributed Acoustic Sensing converts a standard single mode telecoms fibre optic cable into an array of distributed sensors to deliver spatially and temporally rich traffic management information. Using new or existing fibre optic infrastructure as an intelligent traffic sensor allows faster, less disruptive and more economical deployments of traffic management solutions, enabling city authorities to intelligently manage traffic flow and reduce emissions.
\end{abstract}

\section{Introduction}

The urban population of the world has grown rapidly. According to the United Nations, the global urban population increased from 751 million in 1950 to 4.2 billion in 2018. Today, $55 \%$ of the world's population lives in urban areas, a proportion that is expected to increase to $68 \%$ by 2050 . This gradual shift in residence of the human population from rural to urban areas, combined with growth of the world's overall population could add another 2.5 billion people to urban areas by 2050 (United Nations, 2018).

This growing urban population is creating many infrastructure challenges for governments. Not least maintaining the mobility of people around the urban environment. With the rise of the digital age, it has become easier to communicate remotely, but people will still want to travel to meet. This will put increasing pressure on optimising the traffic management systems for the arterial routes into and around urban areas. Distributed Acoustic Sensing makes use of existing or new roadside fibre infrastructure to provide traffic management information which can be used to optimise mobility for road users.

\section{Principles of Distributed Acoustic Sensing}

Distributed Acoustic Sensing (DAS) is a technology that enables continuous, real-time measurements along the entire length of a new or existing, single mode fibre optic cable. The fibre optic cable is transformed into a sensor by an Interrogator Unit plugged onto the end of a single unused spare core. The Interrogator Unit injects a launch pulse of laser light into the optical fibre. As this launch pulse travels along the fibre, interactions within the fibre result in a small amount of light being returned towards the origin. The intensity of this backscattered light is then measured by the Interrogator Unit which, knowing the time of flight, accurately maps backscatter events to fibre distance. The backscatter is modulated by local sound and vibrations around the cable, forming an array of channels spaced every $10 \mathrm{~m}$, listening at audible frequencies and below. Each Interrogator Unit can enable up to $40 \mathrm{~km}$ of fibre optic cable for sensing purposes. This allows vehicles to be detected and tracked as they move alongside the fibre optic cable. This information is then used to provide a number of traffic management applications.

Improvements in the measurement methodology have led to the ability to derive a quantitative phase and amplitude from the received backscatter over shorter lengths of fibre cables for applications utilised today in certain industries (Mateeva et al., 2012). Recent advances have led to the ability to derive quantitative phase and amplitude information over far longer ranges opening possibilities for advanced processing techniques and further applications of the technology.

Figure 1 Outline of DAS
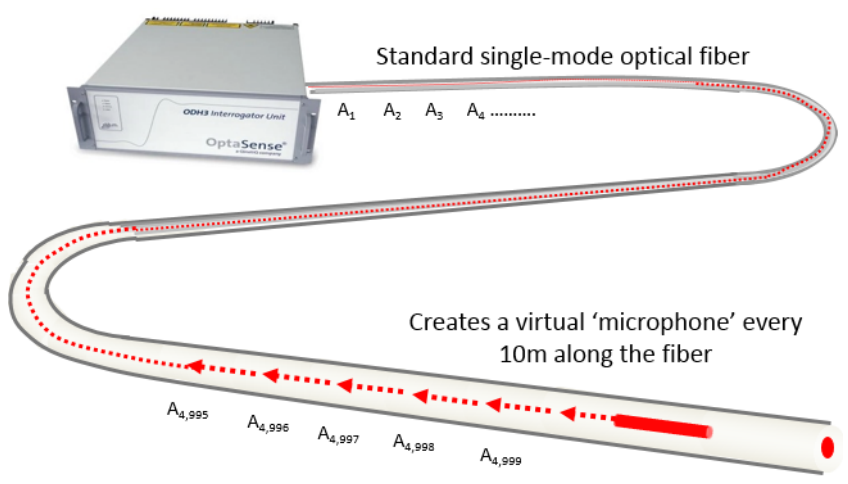


\section{DAS Traffic Management Solution}

\subsection{Ease of installation and accessibility}

By using spare capacity on new or existing roadside fibre optic cables, any single mode fibre optic cable can be quickly enabled into an intelligent traffic management sensor using DAS with minimal fibre work at both ends of the monitored section.

This makes it a compelling solution where traffic management overheads are prohibitive for conventional in road detection sensors that involve new infrastructure to be installed in or around the road, causing expensive road closures and ongoing maintenance periods (Pinchen et al., 2014). The ability to cover an entire route with minimal roadside equipment may also reduce the need for cost vs performance compromises to be made.

Spare capacity is typically available within fibre optic cables due to a mixture of company's future-proofing their networks from exponential data growth combined with technical advances in the way that multiple data signals are transmitted simultaneously over the same fibre cores.

\subsection{Outline of System Architecture}

A DAS deployment comprises of four major elements:

- Sensor - The system utilises conventional telecommunications single mode fibre optic cable as the sensing element. The system needs only a single spare core for fibre sensing.

- Interrogator Unit - The Interrogator Unit connects onto the fibre and provides the sensor interrogation and digitisation of the data. A discrete IU is required for each segment of fibre optic cable that is to be monitored, this could be up to $40 \mathrm{~km}$ fibre length.

- $\quad$ Processing Unit - The Processing Unit takes the feed of data from Interrogator Unit(s) and provides input processing before feeding the data into a powerful suite of classification techniques. These include a mix of signal processing algorithms, machine learning techniques and logic steps which generate valuable traffic management information.

- Data transmission - The output of the classification algorithms, as well as any heartbeats or system health status messages can either be shown on a graphic user interface or sent through a variety of different interfaces to be integrated into other exiting platforms. This could include Variable Signs and Signals (VSS) on road gantries for example.
Figure 2 Outline of typical System Architecture

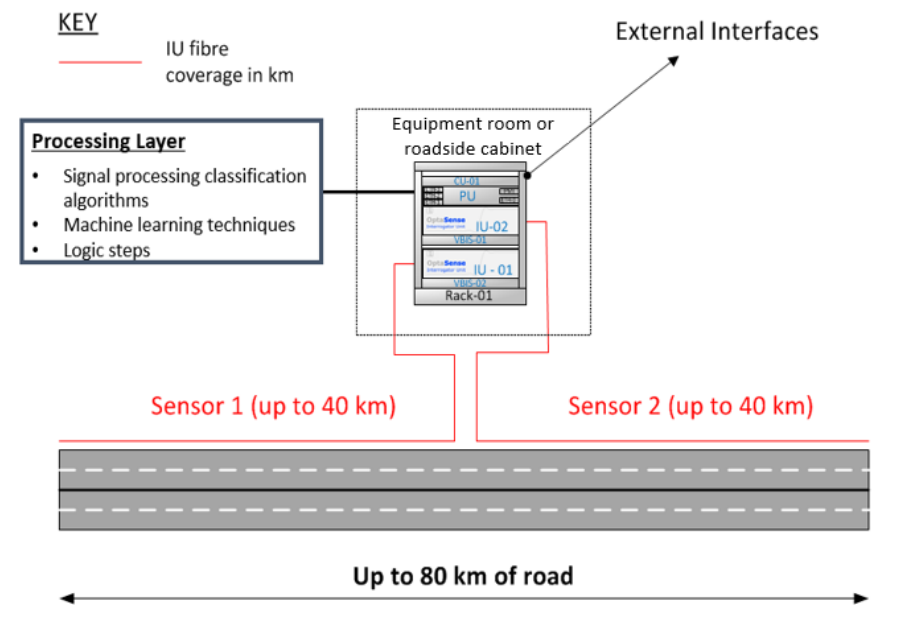

\subsection{Detected signals and sensitivity}

For each $10 \mathrm{~m}$ channel, the intensity of backscattered light is measured. As a road vehicle moves alongside the fibre the sound and vibrations emitted modulate the backscatter and change the intensity of the backscatter. This offers a method for vehicle detection. Figure 3 shows a typical response of a channel over a period of 30 seconds showing multiple vehicles passing the channel location.

As each channel is part of an array, then the neighbouring channels can be used to provide more information. Figure 4 shows two graphs. The top graph details the amplitude (y-axis) against distance along the fibre optic cable (x-axis). The bottom graph displays the amplitudes as a colour, with highlevel amplitudes being yellow and lower levels turning through light blue to dark blue. Time is displayed on the y-axis and distance along the fibre is displayed on the $\mathrm{x}$-axis. The graph shows the tracks of three separate vehicle movements from left to right within the 60 second period. The high-level amplitude in the top left of the top graph is associated with a lorry whilst the lower-level amplitude of the vehicle on the top right of the top graph is a signature from a car.

This array of information allows the classification of vehicle speed to be undertaken and produces distinctive patterns associated with queuing traffic or congestion. By using the information from both individual channels and the array of channels the distinctive patterns of vehicles moving or queues forming parallel to the fibre can be classified and distinguished from other noise sources.

The sensitivity of the system is such that lorries driving parallel to a typical existing buried fibre can be detected at offsets of up to 50 metres, whilst cars can be detected up to 30 metres. 
Figure 3 Individual channel response

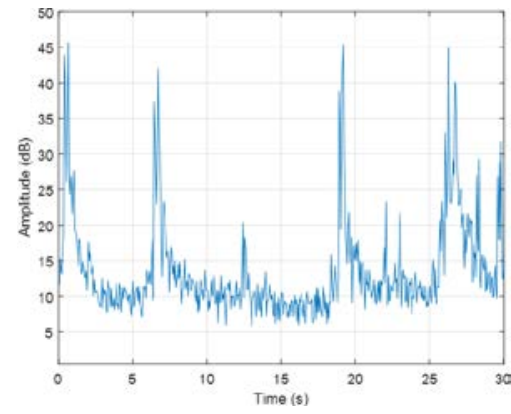

Figure 4 Grouped channel response

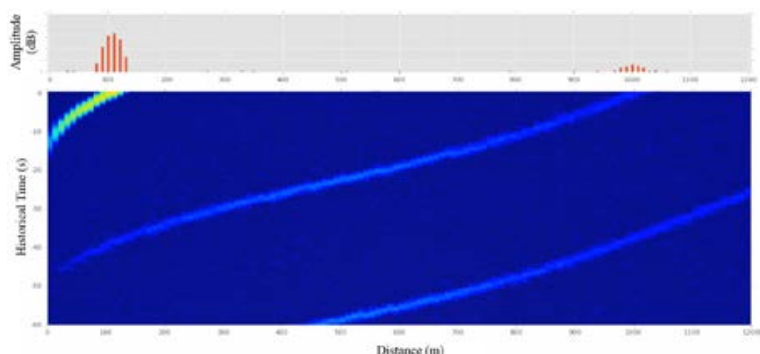

\subsection{DAS Traffic Management Applications}

Traffic Management Applications that can be delivered with DAS are shown in Figure 5. Those applications that can be delivered with a fibre running parallel to the road are detailed in sections 3.4.1 to 3.4.3. Section 3.4.4 details an application that can be delivered where the fibre runs perpendicularly to the road such as a fibre crossing.

3.4.1 Average traffic speed: The cross-sectional traffic speed for each carriageway is calculated for every $10 \mathrm{~m}$ along the monitored section which is then typically averaged to provide outputs every $50 \mathrm{~m}$. This is updated every second and provides a key input into other applications.

Figure 5 Traffic Management Applications

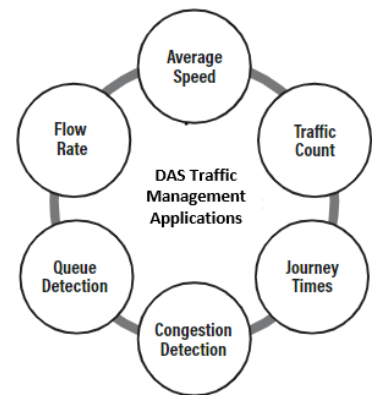

3.4.2 Queue and congestion detections: The sensing system will detect when moving traffic comes to a halt to form a queue and track the queue length. These detections along the fibre array displays distinctive patterns which can be classified. In addition, the system will detect sections of slow moving traffic indicative of congestion. These outputs can interface with Variable Signs and Signals (VSS) on road gantries to provide road users with a wide range of visual warning messages and other upstream traffic information. Within the UK, the purpose of VSS is to meet the Highways Agency's obligations as a network operator which includes incident management, reducing congestion, informing motorists, improving network performance and ensuring the safety of road users and workforce (HA, 2011). On the main arterial roads into and around smart cities the feed of spatially rich, low latency traffic information can optimise the management of incidents and the optimisation of flow.

3.4.3 Journey Times and Route Planning: The spatially and temporally rich speed information can be used for calculation of journey times from any locations along the monitored fibre route. This information can help improve mobility for travellers by planning their optimal route or method of transport.

3.4.4 Traffic count and flow rate: Where the fibre crosses perpendicularly across the road, a traffic count and combined with the average traffic speed, flow rate can be calculated. These could feed into traffic management applications in real time or also provide useful information into the overall planning for future improvements to the road network. Alternatively, using this information, temporary traffic management schemes used for city events can be evaluated and optimised further.

\subsection{Future applications}

Distributed Acoustic Sensing is a platform technology for multiple applications. The number of application within the traffic industry and other industries such as pipeline monitoring are growing. This could lead to other smart city use cases. With the advent of long range quantitative DAS further processing techniques are available leading to the potential for further applications. For near surface monitoring, DAS could be an enabling technology for ambient seismic noise methods using the passive noise from road vehicles (Dou et al, 2017). This could lead to monitoring any potential structural changes within the near surface that could lead to a heightened risk of road surface failure.

\section{Smart Cities}

\subsection{Improving mobile technology}

Mobile technology is constantly evolving to keep pace with growing demand. Each generation of mobile technology has opened up new possibilities that have transformed the way we live and work. The next generation of mobile technology, $5 \mathrm{G}$, is an umbrella term for all the mobile communication technologies required. Unlike previous generations of mobile networks, $5 \mathrm{G}$ is not merely an extension to these but a system of systems. Although technical standards have yet to be finalised, 5G has the clear potential to offer a variety of new and enhanced capabilities over existing mobile technologies. These include higher data rates, lower latency, higher energy efficiency and improved performance in challenging environments (ITU, 2015 and 2017).

\subsection{Role of $5 G$ for smart cities}

Given the complexity of future $5 \mathrm{G}$ systems, it is not yet clear the locations of where $5 \mathrm{G}$ networks will be deployed. The development will likely be interleaved within a wider wireless 
connectivity eco-system. Cities across the globe have smart city strategies in place (Future Cities Catapult, 2017). However, the $5 \mathrm{G}$ mobile technology is seen as a key enabler for a massive urban IoT (Internet of Things) network at the helm of smart city use cases (IHS Markit, 2017). Partly for its ability to integrate with other access technologies to form a continuous network, particularly for urban environments (Palatella et al, 2016).

\subsection{Increased fibre network deployment}

To support future $5 \mathrm{G}$ systems, the UK government is already taking action to drive the increased deployment of fibre. Within the 2016 Autumn Statement, the government announced a $£ 1.1$ billion investment for local roads and local transport (UK GOV, 2016). £200 million will fund a programme of local projects to test ways to accelerate market delivery, and $£ 400$ million, at least matched by private sector investors, will establish a new Digital Infrastructure Investment Fund, to provide developers with greater access to commercial finance. A significant portion of the overall investment is aimed to be used to deliver more extensive full fibre networks. The UK government also introduced a $100 \%$ business rate relief for new full-fibre infrastructure.

The increased fibre network required for enabling $5 \mathrm{G}$ networks will allow a rich network of fibre optic cables that can be transformed into Distributed Acoustic Sensors. Fibre optic cables laid near roads will allow the infrastructure to be enabled for traffic management applications.

\section{Installation Considerations}

Optimum performance for DAS for traffic management solutions is dependent on cable type, installation method, cable depth and position, and the environmental conditions of the site. It is widely applicable for existing fibre installations and allows the potential for optimal installations to be utilised for new fibre infrastructure installations.

\subsection{Cable type}

For traffic management applications, DAS requires good acoustic coupling between the fibre, the cable itself, and the environment which the acoustic events are to be detected whilst being sympathetic to the level of protection or armour necessary to meet local regulations. The generally preferred cable selection for DAS will typically have the following features (FOSA, 2018):

- $\quad$ Single mode

- $\quad$ Tight buffered or gel filled loose tube

- $\quad$ Single jacket

- Unarmoured or single armour

- Spare dark fibre cores (generally one for each $40 \mathrm{~km}$ monitored).

Figure 6 shows tight buffer cable structures with a tactical cable shown on the left and the distribution unit shown on the right. Figure 7 shows loose tube cable structures with an unarmoured single jacket cable construction on the left and a single steel tape armoured cable on the right which can be used for a direct burial installation.
Figure 6 Tight buffer cable structure

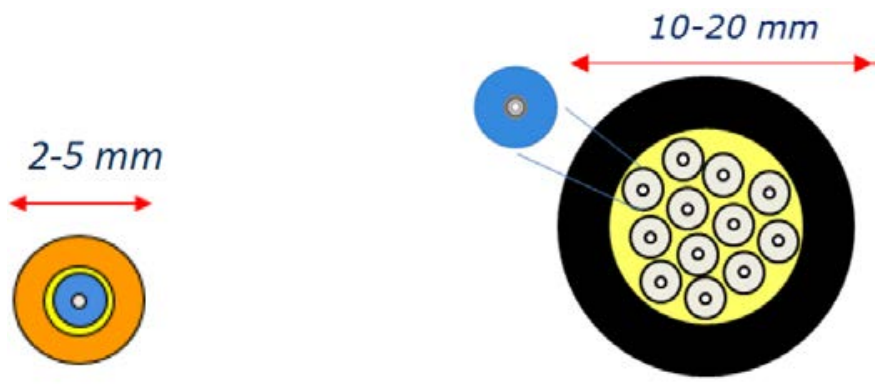

Figure 7 Loose tube cable structure
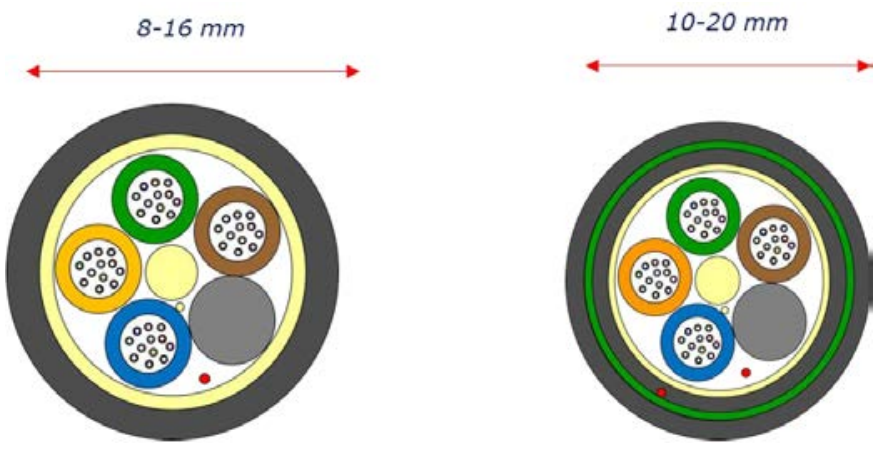

\subsection{Installation method}

The key is finding an installation method that places the cable close to the edge of the monitored road whilst protecting the cable from damage, without unduly reducing sensitivity. Four typical installations are now detailed and listed in order of most to least recommended.

5.2.1 Slot cut fibre cable: Slot cut fibre gives direct acoustic coupling between the fibre and the traffic roadway.

Figure 8 Slot cut deployed fibre cable

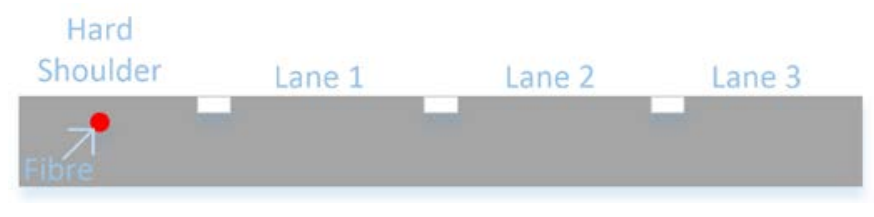

5.2.2 Directly buried fibre cable: Directly buried fibres, usually found at the edge of the road where the fibre cannot be slot cut into the hard shoulder, gives acoustic coupling with the ground. This method minimises the number of ground medium boundaries (road and soil) between the fibre and the traffic and therefore achieves good performance.

Figure 9 Directly buried fibre cable

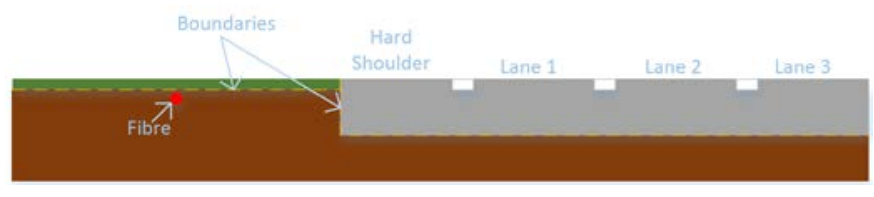

5.2.3 Directly buried ducted fibre cable: A duct will add to reduce the level of signal accessible on the fibre. However, a 
non-armoured cable deployed in ducting will deliver a similar order of magnitude of performance to an armoured cable directly buried. The amount of reduction in the sensitivity is dependent on the type of ducting.

Figure 10 Directly buried ducted fibre cable

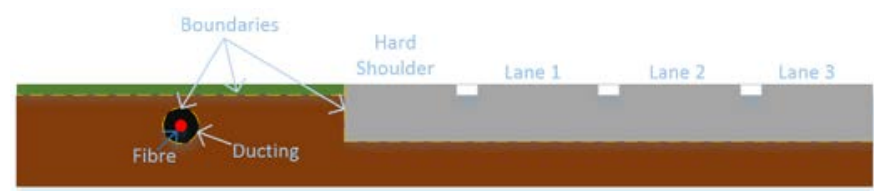

5.2.4 Troughed fibre cable: Fibres that are located within a concrete trough will achieve less sensitivity than the other deployment types. However, troughed fibre can be used for traffic management applications.

Figure 11 Fibre cable in a concrete trough

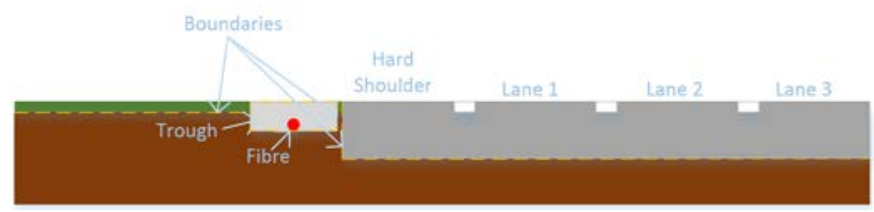

\subsection{Cable depth}

The optimum cable depth for burying the fibre is dependent on the burial method. Generally, the deeper the installation the further away from the road surface and so a further reduction in sensitivity. Each suggested burial depth for the respective methods are tabulated in Table 1.

Table 1 Recommended fibre burial depths

\begin{tabular}{ll}
\hline Burial method & Depth (in cm) \\
\hline Slot Cut & $10-30$ \\
Direct buried (single armour) & $30-50$ \\
Conduit & $30-50$ \\
Troughed & $20-40$ \\
\hline
\end{tabular}

\subsection{Fibre cable offset distance}

DAS senses acoustic signals from a range of distances across the asset. Generally, the further the fibre is located from the noise source the smaller the signal received. The main factor to take into account when placing the fibre is the physical distance from the lane(s) that need to be monitored. It is beneficial to locate the fibre installation near to, but not within, the active lanes to ensure the fibre is easily accessible.

DAS traffic management and mobility algorithms require a certain amount of signal to work effectively. If the fibre offset from the first lane is greater than the recommendations found in Table 2 there will likely be a reduced system performance due to fewer detected vehicles being tracked.
Table 2 Recommended fibre offset

\begin{tabular}{lll}
\hline Burial Method & $\begin{array}{l}\text { Offset from lane } \\
\text { (in m) }\end{array}$ & Performance \\
\hline & $<10$ & Very Good \\
Slot Cut & $10-14$ & Good \\
& $14-17$ & Average \\
& $<9$ & Very Good \\
Direct buried $^{\mathrm{a}}$ & $9-13$ & Good \\
& $13-16$ & Average \\
& $<8$ & Very Good \\
Conduit & $8-12$ & Good \\
& $12-15$ & Average \\
Troughed & $<7$ & Good \\
& $7-12$ & Average
\end{tabular}

${ }^{\mathrm{a}}$ Direct buried single armoured fibre

\subsection{Fibre layout}

An optimum DAS installation for divided roadways is with cables on each side of the carriageway. This accounts for any elevation changes between the different roadways which with a single fibre would result in reduced performance and in extreme height differences of greater than $5 \mathrm{~m}$, a complete loss of vehicle detection from the far carriageway. Within the urban environment raised roads with separate superstructures for each carriageway can be a common occurrence and would need to be monitored with separate fibres.

Figure 12 Fibre deployment on both sides
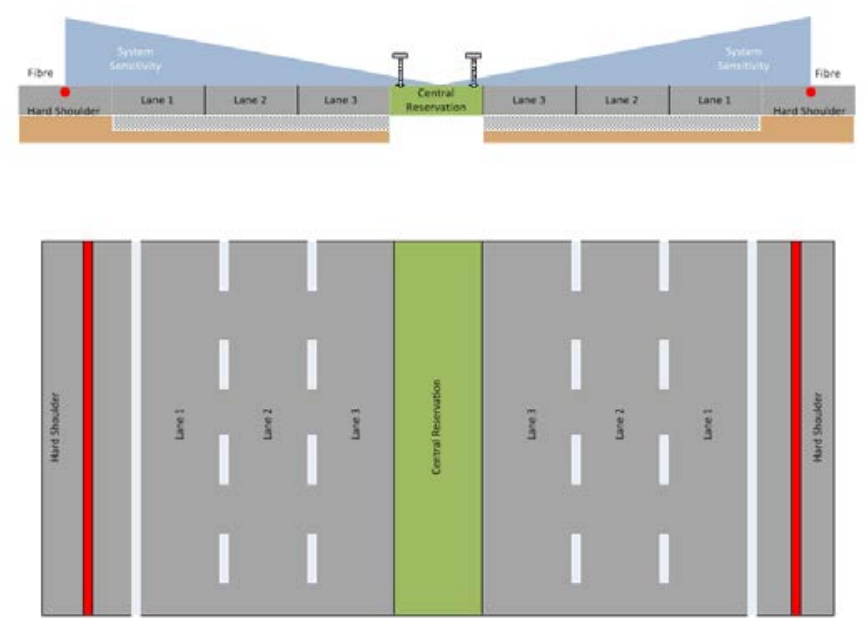

Where central reservations are narrow, the carriages don't separate or split into different elevations, deployments within the central reservation offers the possibility of monitoring both sides of a road with a single fibre. 
Figure 13 Central reservation fibre deployment
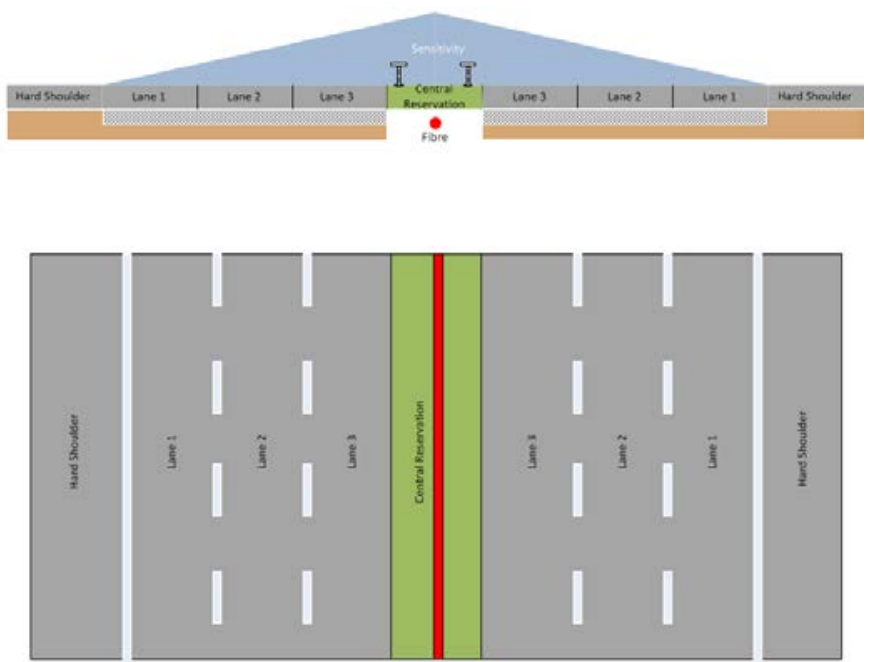

Fibre deployment options for tunnels and bridges are more limited than for ground surface-based sections. However, the slot cut options and troughed fibre deployments can be used for these infrastructure types.

At certain locations along the monitored road, a fibre optic cable that is installed perpendicularly can be utilised for counting traffic. The vehicles crossing the fibre can be detected and counted. Although a perpendicular fibre is only suitable for counting traffic, it can be integrated into a parallel running fibre system to achieve all applications.

To accurately distinguish the bi-directional traffic flow, an additional length of fibre should be located between the carriageways to provide signal isolation. Commonly this takes the form of a fibre spool $(>50 \mathrm{~m})$ in the central reservation or, if applicable, a staggered fibre crossing as shown in Figures 14 and 15 , respectively.

Figure 14 Fibre crossing with spool

T I
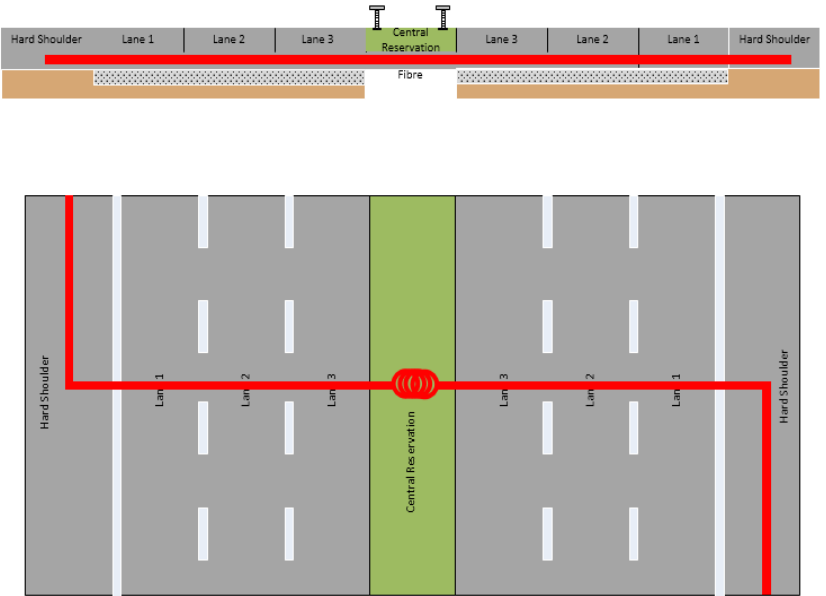

Figure 15 Staggered fibre crossing
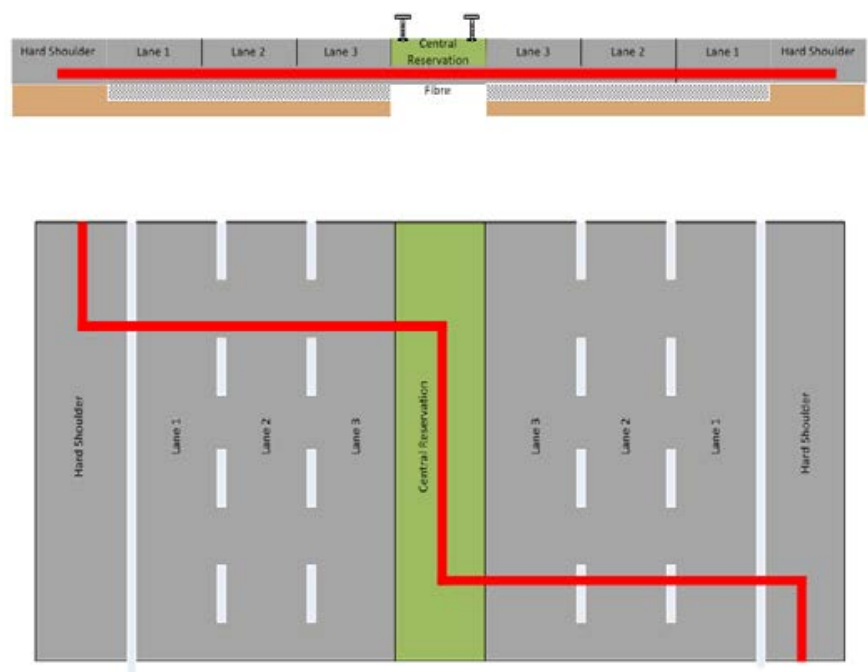

\section{Conclusion}

The 'smart city' concept will utilise advancements in technology and data to plan and operate cities better. For traffic management, the opportunities that these advancements afford are coupled with the challenges of a growing global urban population. A central challenge will be maintaining the mobility of people around cities. Distributed Acoustic Sensing can enable existing or new roadside fibre optic cables to be converted into intelligent, distributed sensors which deliver traffic management information for traffic managers and road users, and mobility information for travellers planning their journey.

On the main arterial roads into and around smart cities the feed of spatially rich, low latency incident detection and congestion traffic information from fibre optic sensors could help optimise the management of incidents and the optimisation of flow, helping to reduce emissions. Additionally, the speed information could be used to calculate journey times between any locations along the monitored fibre sections, helping the mobility of travellers by planning their optimal route or method of transport.

With the advent of 5G mobile technology as a key enabler for an urban IoT (Internet of Things) network at the helm of the smart city use cases, there will be the need for significant further fibre infrastructure, creating to further opportunities for enabling distributed traffic management sensors. With ongoing advances in the technology, the rise of long range quantitative DAS sensors will allow the possibility for new applications to be deployed. By installing the fibre infrastructure in the right place, the possibility is there to transform the fibre into a distributed sensor for existing traffic management applications or for future quantitative DAS applications.

\section{Acknowledgements}

Thanks to the Fiber Optic Sensing Association (FOSA) for the use of Figures 3 and 4. 


\section{References}

\section{Journal articles}

Mateeva, A et al. (2012) Advances in Distributed Acoustic Sensing (DAS) for VSP. Society of Exploration Geophysicists. SEG-2012-0739.

Dou, S et al. (2017) Distributed Acoustic Sensing for Seismic Monitoring of The Near Surface: A Traffic-Noise Interferometry Case Study. Nature, Scientific Reports 7. Article number 11620 .

Palattella et al. (2016) Internet of Things in the 5G era: Enablers, Architecture, and Business Models. Institute of Electrical and Electronics Engineers Journal on Selected Areas in Communications 34 (3), 10.1109/JSAC.2016.2525418.

\section{Conference paper}

Pinchen, R, Wu, H and Swift-Hoadley, D (2014) Congestion and Incident Management Applications for Distributed Acoustic Sensing Technology. New Zealand Transport Agency in conjunction with the Welsh Transport Agency. ITS Asia-Pacific 2014 Forum.

\section{Reports}

United Nations (2018) Revision of World Urbanization Prospects. Department of Economic and Social Affairs, United Nations Headquarters, New York, NY 10017, USA.

Highways Agency (2011) Highways Agency policy for the use of Variable Signs and Signals (VSS). Highways England, Bridge House, 1 Walnut Tree Close, Guildford, GU1 4LZ, UK.
ITU, International Telecommunication Union (2015) IMT Vision - Framework and overall objectives of the future development of IMT for 2020 and beyond. International Telecommunication Union, Place des Nations, 1202 Geneva, Switzerland.

ITU, International Telecommunication Union (2017) Minimum requirements related to technical performance for IMT-2020 radio interface(s). International Telecommunication Union, Place des Nations, 1202 Geneva, Switzerland.

Future Cities Catapult (2017) Smart City Strategies: A Global Review. Urban Innovation Centre, 1 Sekforde Street, London, EC1R 0BE, UK.

IHS Markit (2017) The 5G Economy: How $5 G$ Technology will contribute to the global economy'. Commissioned by Qualcomm. IHS Markit Global Headquarters, $4^{\text {th }}$ floor Ropemaker Place, 25 Ropemaker Street, London, EC2Y 9LY, UK.

FOSA. Fiber Optic Sensing Association (2018) Installation considerations for rail. Headquarters, 2025 M Street NW, Suite 800, Washington, DC 20036, USA.

\section{Websites}

UK GOV (2016). Autumn Statement 2016 transport announcements. [online] Available at: https://www.gov.uk/government/publications/autumnstatement-2016-transport-announcements/ [accessed 05/01/2019] 